Marc Kupietz/Nils Diewald/Eliza Margaretha/Franck Bodmer/ Helge Stallkamp/Peter Harders (Mannheim)

\title{
Recherche in Social-Media-Korpora mit KorAP
}

\section{Einleitung}

Die Korpusanalyseplattform KorAP ${ }^{1}$ wird als Nachfolgesystem zu COSMAS II am Leibniz-Institut für Deutsche Sprache (IDS) entwickelt und erlaubt einen umfassenden Zugriff auf einen Teil von DEREKo (Kupietz et al. 2010). Trotz einiger noch fehlender Funktionalitäten ist KorAP bereits produktiv einsetzbar. Im Folgenden wollen wir am Beispiel der Untersuchung von Social-Media-Korpora einige neue Möglichkeiten und Besonderheiten vorstellen.

\section{Erstellung eines virtuellen Korpus}

Vor allem wenn es darum geht, eine bestimmte Sprachdomäne wie Social-MediaKommunikation auf Grundlage eines universellen Korpus zu untersuchen, ist die Möglichkeit der Bildung spezieller Sub-Korpora essenziell. In KorAP können solche sogenannten virtuellen Korpora (Kupietz et al. 2010, S. 1849) frei auf Basis indizierter, dokumentbezogener Metadatenfelder definiert werden (Bański et al. 2013). So lässt sich eine Suche auf Dokumente beschränken, die etwa einem bestimmten Texttyp zugeordnet sind oder von einem bestimmten Autor oder einer bestimmten Autorin verfasst wurden. Virtuelle Korpora, die nach komplexeren Kriterien erstellt werden müssen, um beispielsweise eine gewisse Größe oder eine Balanciertheit in Bezug auf eine Domäne aufzuweisen, lassen sich extern definieren und als hinterlegte virtuelle Korpora nach dem Vorbild von COSMAS II referenzieren (siehe Abb. 1; vgl. Kupietz et al. i. Ersch.). Diese referenzierten Korpora können zusätzlich Teil komplexerer virtueller Korpora werden - so lassen sich mehrere hinterlegte virtuelle Korpora kombinieren und unter Einbeziehung zusätzlicher Metadatenkriterien weiter einschränken.

1 https://korap.ids-mannheim.de/ (Stand: 24.9.2019). 


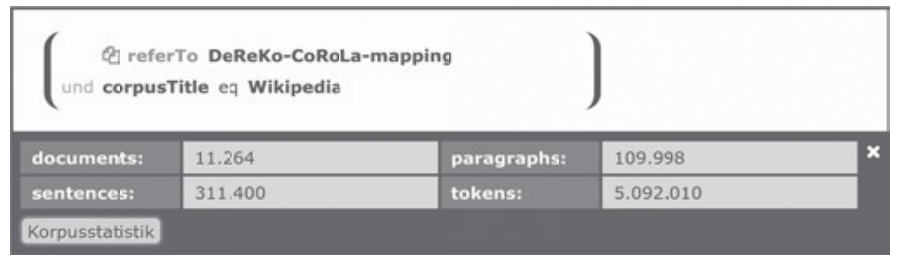

Abb. 1: Virtuelles Korpus aus allen Wikipedia-Dokumenten, die gleichzeitig Teil eines hinterlegten virtuellen Korpus ${ }^{2}$ sind

Um die Definition virtueller Korpora zu vereinfachen, insbesondere, wenn dem Nutzer oder der Nutzerin die unterschiedlichen Werte der Metadatenfelder nicht bekannt sind, können diese mithilfe des „Corpus by Match“-Assistenten erstellt werden. Dabei kann durch Auswahl von Werten aus der Metadatenansicht eines bestimmten Treffers einem definierten virtuellen Korpus diese Werte als weitere Bedingungen hinzugefügt werden (siehe Abb. 2). Ähnlich dem „Query by Match“Assistenten (Kupietz et al. 2018) werden die neuen Kriterien zunächst angezeigt, bevor sie durch ein bestätigendes Klicken dem virtuellen Korpus hinzugefügt werden. Dies soll zum einen Fehler bei der Konstruktion des virtuellen Korpus vermeiden, zum anderen die Erstellung so transparent wie möglich machen.

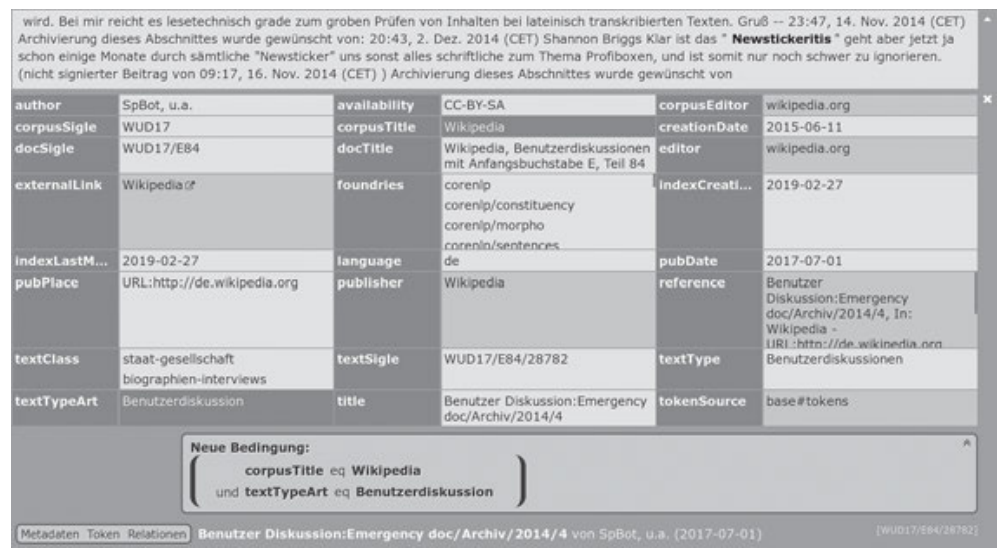

Abb. 2: Der „Corpus by Match“-Assistent. Konstruktion eines virtuellen Korpus aus allen Texten mit der Korpus-Zugehörigkeit „Wikipedia“, die dem Texttyp „Benutzerdiskussion“ zugeordnet sind, anhand der Metadaten eines Treffers zum Suchwort „Newstickeritis“.

2 In diesem Fall ein Subkorpus aus DeReKo, das mit einem Subkorpus des rumänischen Nationalkorpus CoRoLa vergleichbar ist (siehe Kupietz et al. i. Ersch.). 


\section{Hypermediale Einbindung}

Social-Media-Korpora weisen, wie alle Sammlungen hypermedialer Texte, eine Vielzahl verlinkter Ressourcen auf. Um diese für die Auswertung unter Umständen relevanter Informationen in KorAP abzubilden, wird die Hinterlegung beliebiger Ressourcen-Typen als Metadaten unterstützt. Diese Ressourcen können zwar abgefragt werden (per Web-API oder eingebettet in das Nutzerinterface), aber nicht Teil der Definition eines virtuellen Korpus werden.

Jeder Ressource können mehrere zusätzliche Meta-Informationen beigefügt werden (modelliert als Parameter einer Data-URI ${ }^{3}$ ), auf deren Basis die Einbindung in das Nutzerinterface vorgenommen wird. Für die Wikipedia-Korpora wurden dabei zunächst die Quellen-URLs als externe Links eingebunden (siehe Abb. 3). So ist es möglich von einem Treffer in KorAP direkt zu der betreffenden Seite in der deutschen Wikipedia zu gelangen. Dies gilt sowohl für Artikelseiten, als auch für Nutzer- und Diskussionsseiten. Neben externen Links wird auch die Hinterlegung beliebiger Textdaten unterstützt, was Meta-Informationen erlaubt, die über das IDS-Textmodell (Lüngen/Sperberg-McQueen 2012) hinausgehen. In Zukunft planen wir, diesen Mechanismus auch für die Verlinkung weiterer Ressourcen zu nutzen, etwa auf Verfasser-Seiten von Social-Media-Beiträgen, oder auch für die Einbindung von Bild- und Tondokumenten.
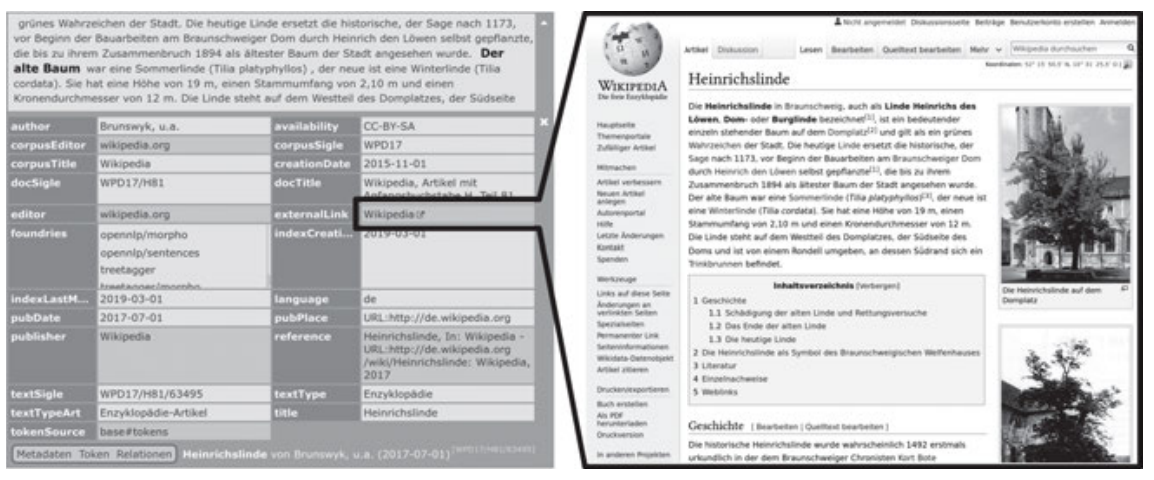

Abb. 3: Einbindung beliebiger Ressourcen in Dokument-Metadaten

Um im Gegenzug Verlinkungen aus externen Applikationen zu ermöglichen, ist die Suche in KorAP zustandslos umgesetzt; das bedeutet, dass eine URL in KorAP alle

3 https://tools.ietf.org/html/rfc2397 (Stand: 24.9.2019). 
relevanten Such-Parameter enthält, und so durch Weitergabe der URL ein Suchergebnis geteilt und verlinkt werden kann. Die Möglichkeit aus externen Anwendungen heraus KorAP-Recherchen auszulösen, wird beispielsweise vom CCDBNachfolgesystem (Arbeitstitel „DEREKo-Vecs“, siehe Lüngen/Kupietz in diesem Band) genutzt, um aus aggregierten Visualisierungen heraus gezielt die dem jeweiligen visualisierten Element zugrundeliegenden Konkordanzen aus einem bestimmten virtuellen Korpus anzeigen zu können. Auf diese Weise können das Zustandekommen der Visualisierung nachvollzogen und etwaige neue Hypothesen direkt überprüft werden. Die Funktionalität ist jedoch nicht auf WebApplikationen beschränkt. Sie wird auch von der Android-VR-App IDS-Wortraumstation $^{4}$ verwendet, um in eingebetteten Web-Views die der 3D-Visualisierung zugrundeliegenden Konkordanzen innerhalb der virtuellen Realität darzustellen (siehe Abb. 4).

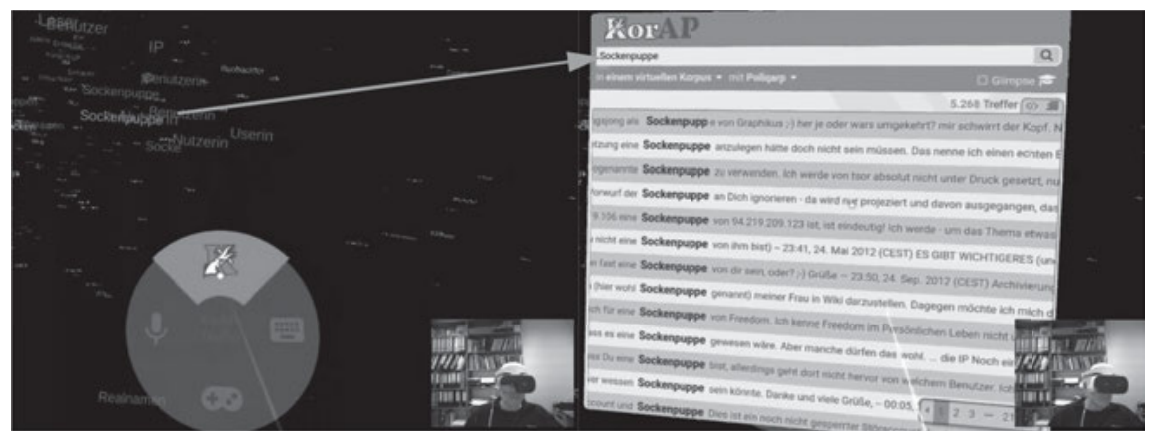

Abb. 4: Anzeige von Konkordanzen zu „Sockenpuppe“ in den Wikipedia-Diskussionskorpora innerhalb der Android-VR-App IDS-Wortraumstation

\section{Guided Tour}

Ein schneller Einstieg in die Recherche mit KorAP in Social-Media-Korpora gelingt mit der neu entwickelten Guided Tour (siehe Abb. 5), die den Nutzer oder die Nutzerin anhand einer exemplarischen Suchanfrage durch das Programm führt. Dabei wird Schritt für Schritt die Erstellung einer solchen Anfrage erklärt und mittels Einblendungen die Funktionen erläutert, die dabei unterstützen.

4 Siehe IDS aktuell 4/2018 unter http://www1.ids-mannheim.de/fileadmin/aktuell/newsletter/ pdf/IDS_aktuell_2018_4.pdf (Stand: 24.9.2019). 
Die Benutzeroberfläche von KorAP ist mit dem Ziel konzipiert, sowohl Anfänger/innen als auch Expert/innen mit unterschiedlichen Forschungsinteressen einen einfachen Zugriff auf heterogene Korpora zu ermöglichen (Diewald/Barbu/ Verginica i. Ersch.). Die Suchanfrage wird, ähnlich wie bei Internetsuchmaschinen, unmittelbar eingegeben, komplexe Funktionalitäten und Informationen werden erst sichtbar, wenn sie gebraucht werden.

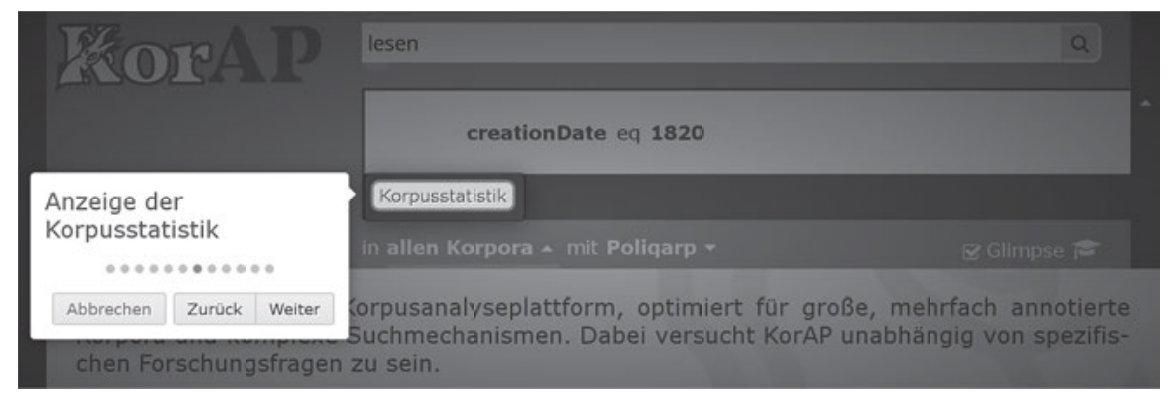

Abb. 5: Guided Tour

Die Guided Tour soll dazu dienen, ohne großen Einarbeitungsaufwand auch komplexere Funktionalitäten nicht zu übersehen.

\section{Literatur}

Bański, Piotr/Frick, Elena/Hanl, Michael/Kupietz, Marc/Schnober, Carsten/Witt, Andreas (2013): Robust corpus architecture: A new look at virtual collections and data access. In: Hardie, Andrew/Love, Robbie (Hg.): Corpus Linguistics 2013. Abstract book. Lancaster, S. 23-25. Internet: http://nbn-resolving.de/urn:nbn:de:bsz:mh39-44855 (Stand: 17.10.2019)

Diewald, Nils/Barbu Mititelu, Verginica/Kupietz, Marc (i. Ersch.): The KorAP interface. Accessing CoRoLa via KorAP. In: Revue Roumaine de Linguistique.

Kupietz, Marc/Diewald, Nils/Margaretha, Eliza/Bodmer, Franck/Stallkamp, Helge/Harders, Peter (2018): Neues von KorAP. In: Eichinger, Ludwig M./Plewnia, Albrecht(Hg.): Neues vom heutigen Deutsch. Empirisch - Methodisch - Theoretisch. (= Jahrbuch des Instituts für Deutsche Sprache 2018). Berlin/Boston, S. 345-349.

Kupietz, Marc/Diewald, Nils/Trawiński, Beata/Cosma, Ruxandra/Cristea, Dan/Tufiş, Dan/Váradi, Tamás/Wöllstein, Angelika (i. Ersch.): Recent developments in the European Reference Corpus EuReCo. In: Granger, Sylviane/Lefer, Marie-Aude/Aguiar de Souza Penha Marion, Laura (Hg.): Book of abstracts. Using Corpora in Contrastive and Translation Studies Conference (5th edition). (= CECL Papers 1). Louvain-la-Neuve, S. 101-103. 
Kupietz, Marc/Belica, Cyril/Keibel, Holger/Witt, Andreas (2010): The German Reference Corpus DEREKo: A primordial sample for linguistic research. In: Calzolari, Nicoletta/Choukri, Khalid/ Maegaard, Bente/Mariani, Joseph/Odijk, Jan/Piperidis, Stelios/Rosner, Mike/Tapias, Daniel (Hg.): Proceedings of the Seventh International Conference on Language Resources and Evaluation (LREC 2010). Paris/Valletta, S. 1848-1854.

Lüngen, Harald/Sperberg-McQueen, Christopher M. (2012): A TEI P5 document grammar for the IDS text model. In: Journal of the Text Encoding Initiative 3, S. 1-18. Internet: https:// journals.openedition.org/jtei/508 (Stand: 24.9.2019). 